\title{
Capabilities of Ultrasound for Monitoring and Quantitative Analysis of Polyolefin Waste Particles in Magnetic Density Separation (MDS)
}

\author{
M.C.M. Bakker* and S.A. Sanaee
}

CITG, Recycling Engineering, Delft University of Technology, Stevinweg 1, 2628 CN, The Netherlands

\begin{abstract}
A study was conducted into the potential of ultrasound as a qualitative and quantitative tool for the Magnetic Density Separator (MDS), where an ultrasound sensor array will operate from inside the MDS ferrofluid. The desired applications of the ultrasound are monitoring of polyolefin particles in flow during the separation process, and performing throughput measurements and quality inspection. The possibility to adapt off-the-shelf medical imaging technology was investigated with an eye towards the real-time requirements for industrial application. It is shown that a medical imager can be adapted for monitoring the flowing particles at speeds up to $30 \mathrm{~cm} / \mathrm{s}$, and the imaging quality can be very good under optimum viewing angle conditions. Ultrasound imaging may also allow for quantitative through-put analysis provided the flowing particles are sufficiently spaced, which puts a limit to the maximum measurable throughput. If viewing conditions are not optimum the medical imager relies on the human operator to improve image quality. This situation is undesired for industrial operation where there may be little or no time for operator intervention. To anticipate these occurrences a second imaging method is proposed that could act in conjunction, which is effective since it is based on a different physical principle. Ultrasound quality inspection is a very different technique, and its feasibility depends first of all on whether different types of polyolefin are acoustically distinctive. Using experiments and 3D acoustic computer modelling to determine wave speed and material attenuation, the potential of ultrasound for polyolefin identification under immersed conditions was investigated. With a calibrated measurement setup it is shown that polyolefins may potentially be classified into acoustically distinctive groups. The possible errors in throughput measurement and quality inspection and the requirements for these techniques to perform under operational MDS conditions are also addressed.
\end{abstract}

Keywords: Waste plastics, polyolefins, magnetic density separation, ultrasound monitoring.

\section{INTRODUCTION}

The European consumption of virgin plastic materials has steadily increased over the last decade. A third of these plastics are polyolefins that are quite suitable for recycling. The marketing value of recycled polyolefins may be increased if they can be separated into pure forms. Since polyolefins are quite close in mass density, new technologies are currently being developed to enable both technical and economical separation. One such development is the Magnetic Density Separator (MDS) for plastics from car shredder residue and household waste [1]. In MDS, a suspension of water and ferrous nano-particles acts as the separating medium. By applying a strong magnetic field from the top, the ferrofluid effectively causes a gradient in mass density ranging from low at the top $\left(<0.85 \mathrm{gcm}^{-3}\right)$ to the normal density of water at the bottom [2]. Plastic particles are inserted at one end into a channel of flowing magnetised ferrofluid. The particles separate and reach a steady position at the depth where their mass density complies locally with that of the fluid, after which they can be easily extracted at the other end of the channel. To monitor and optimise the MDS process the particles must be observed and studied under operational conditions.

*Address correspondence to this author at the CITG, Recycling Engineering, Delft University of Technology, Stevinweg 1, $2628 \mathrm{CN}$, The Netherlands; Tel: +31 (0)15 27 85219; E-mail: M.C.M.Bakker@TUDelft.nl
Unfortunately, visual and infrared observation systems are rendered useless since the preferred ferrofluid is opaque, essentially black. This has lead to the present study into ultrasound as a promising technique for monitoring and quantitative analysis.

The ferrofluid flow speed during full MDS operation is around $30 \mathrm{~cm} / \mathrm{s}$, which presents a challenge for an on-line ultrasound system to visualize the plastic particles in realtime. Since medical ultrasound imaging systems are wellestablished commercial technology they give a point of departure for the technology development for industrial MDS. A point of attention is that medical imaging systems are obviously optimized for human tissues and dedicated to usage by medical doctors, which is why their suitability for adaptation to industrial usage has to be investigated first. The main research in the medical ultrasound field is towards 3D (3D space+time) imaging systems. However, these systems require much more data and achieve lower image rates and are also considerably more expensive than $2 \mathrm{D}$ systems $(2 \mathrm{D}$ space+time). Therefore a state-of-the-art 2D system was used in the imaging tests, which was capable of scanning at least 25 images per second. These tests were aimed at the shape consistency, clearness and sharpness of the images for both static objects and particles moving within the ferrofluid.

The excellent performance of medical imagers is largely due to the efficient method of data scanning and straightforward physical interpretation of the signals [3]. This performance depends on the probe orientation and machine 
settings, so if the monitoring conditions change this means the operator must intervene. This gave some reason for concern in view of the desired continuous real-time capability of industrial MDS monitoring. As a potential backup, the capability of the Synthetic Aperture Focusing Technique (SAFT) was tested [4]. SAFT relies on a different physical principle and is successfully employed for detecting material flaws in areas of non-destructive testing [5]. If required, the SAFT principle could be combined with the medical imaging principle in a single system to take over in cases of sudden changes in image quality.

Throughput measurement serves as a monitoring of the economical performance of the MDS. It is measured as the total weight or volume of particles that is output by the MDS per unit of time. To perform throughput measurement by ultrasound the volume of each individual particle must be estimated from the images in real-time. The requirements to this technique are therefore basically the same as for monitoring. The accuracy of an individual particle volume measurement may be relaxed thanks to the large amounts of particles involved in MDS polyolefins separation, where economical throughout can be measured in tons per hour [2]. On the other hand, the measurable amount of particles will be limited by the sensitivity, size, and range of the sensor array. Therefore the requirements for economical MDS throughput measurement need to be established and related to properties such as error, performance, and capacity.

Quality inspection is required because different polyolefins may have the same mass density, in which case a density-based technology like MDS would deliver them in the same output fraction. A prime example is polypropylene (PP) and low-density polyethylene (LDPE). Inspection requires detection of material properties of individual plastic particles, after which each particle can be identified as belonging to a specific group of polyolefin. This task will be particularly challenging because detection has to be carried out in real-time from inside the ferrofluid of the MDS. This will offset it from common practice where quality inspection is applied to either the input or output streams of a waste separator [6,7]. The potential of ultrasound for quality inspection is investigated by determining if the targeted polyolefin groups are acoustically distinctive. To that end the acoustic wave speeds of various polyolefin samples are measured in a calibrated set-up. The acoustic material attenuation is characterized by a relaxation time that is accurately determined by matching a 3D model for acoustic wave propagation to the experimental data. Eventually, quality inspection will have to be carried out under operational MDS conditions. Therefore, just as for throughput, requirements for economical MDS quality inspection need to be established and related to properties such as errors, performance, and capacity.

\section{ULTRASOUND MONITORING}

\subsection{Array Principle and Sensitivity}

In ultrasound imaging a piezoelectric sensor array generates and receives wave pulses that are returned by scattering and reflecting objects. When acoustic waves encounter an object, or generally any change in material properties, they either reflect or scatter depending on the roughness of the surface. Some of these waves return to the sensor array, now acting as a receiver, where the amplitude is logged as a function of time. After processing the data the reflecting and scattering surfaces can be reconstructed at the correct distance from the sensor to give a $2 \mathrm{D}$ picture of the interior of the acoustic medium at hand [3]. A line array of many small piezoelectric sensors is used to obtain sufficient spatial resolution and width of the reconstructed image. In the medical imaging technique, the waves of interest propagate in a more or less straight beam that is typical for the sensitivity of a grouping of small sensor elements. The wave beam direction may be electronically manipulated in the length direction of the array by modifying time delays in both firing and receiving of neighbouring sensor elements. The beam width in the direction orthogonal to the array is determined solely by the physical array design and is quite narrow, up to a few millimetres wide. On the whole the wave beam properties effectively create ultrasound sensitivity in a flat sheet of space in front of the array. A typical sensor array for medical imagers is $35 \mathrm{~mm}$ wide and allows imaging up to $120 \mathrm{~mm}$ distance from the array. Dependent on the angle of scanning with respect to the orientation of the object this may give images that can be suggestive of cross-sections like those produced in X-ray computer tomography (CT). Unlike CT, ultrasound images dominantly contain surface features that were visible in direct view of the array and inside the ultrasound beam.

\subsection{Medical Imager Capability}

The medical imaging algorithms are designed for human tissues, which acoustic properties are close to that of water [8]. Plastics are generally more reflective and attenuating, and the possible influence of the ferrofluid [9] on image quality should also be investigated. Fig. (1) shows the experimental set-up for capturing ultrasound images of moving plastic particles inside ferrofluid. Three typical particles of different shapes and materials from car shredder residue are attached to a thin string that is wrapped over two wooden rolls. The speed and motions of particles in a MDS is approximated by simply rotating the rolls from above the fluid by another string (not shown). The particles were 10-20 $\mathrm{mm}$ long, $5-10 \mathrm{~mm}$ wide and $2 \mathrm{~mm}$ thick. The tests were carried out using a commercial ultrasonic imaging system. The linear array had $35 \mathrm{~mm}$ aperture with 128 elements and was excited by pulses in the bandwidth of 3-11 MHz. The available observation range was $1 \mathrm{~mm}$ to $120 \mathrm{~mm}$ from the array.

Fig. (2) shows images captured from a movie sequence of the triangular plastic particle shown on the left in Fig. (1). The particle is imaged under both static conditions and when it was moved up to $30 \mathrm{~cm} / \mathrm{s}$. The other particles gave images of comparable quality. In both cases the shape of the particle is recognizable, clear, and relatively sharp. The smallest visible details are about $0.5 \mathrm{~mm}$. The capability of the medical imager for detecting small details from surface scattering in real-time is also clear from the thin string to which the particle was attached. It is noted that the orientation of the sensor array was different for the moving particle image.

The good shape consistency and sharpness of ultrasound images is also demonstrated in Fig. (3) using a M13 bolt and screw thread because they present easily identifiable shapes. 


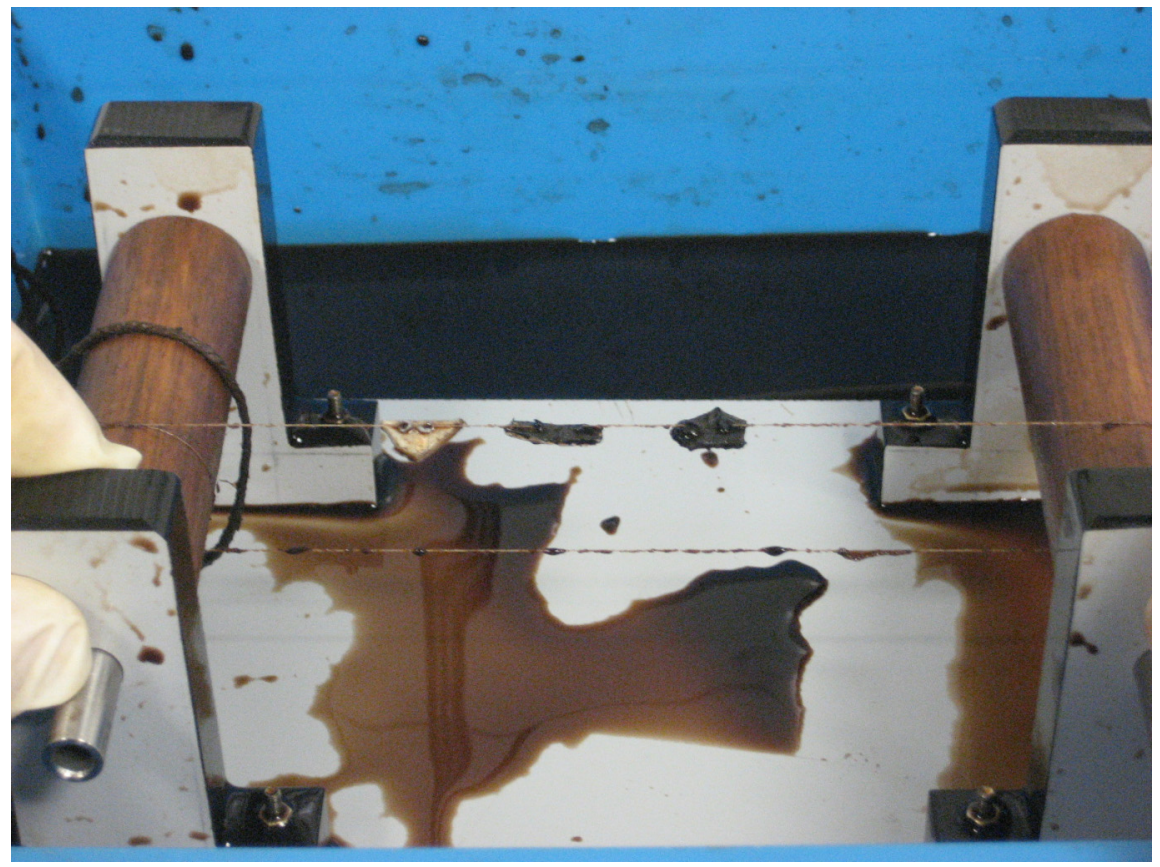

Fig. (1). Experimental set-up for simulation of moving particles in the black ferrofluid.
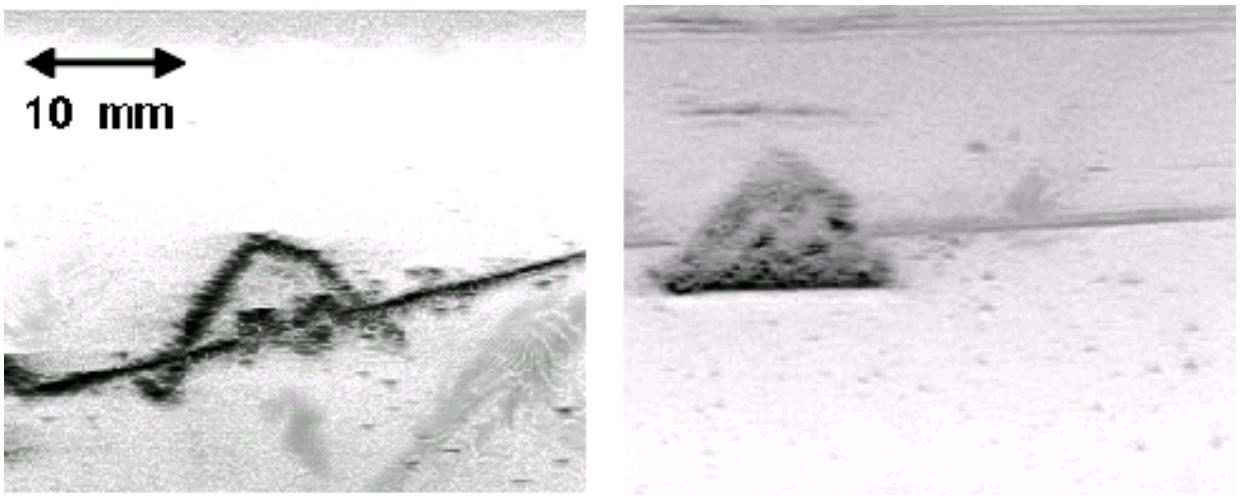

Fig. (2). Images of a small particle in ferrofluid. Left panel: static. Right panel: moving at $30 \mathrm{~cm} / \mathrm{s}$.

The left panel shows the image when the whole underside of the bolt (no thread here) is irradiated from the left, which requires that the object is at a slight angle to the wave beam. This picture could easily be mistaken for a frontal view. The right panel in Fig. (3) shows a cross section of the top of the bolt, also for left-side irradiation and under a slight angle.

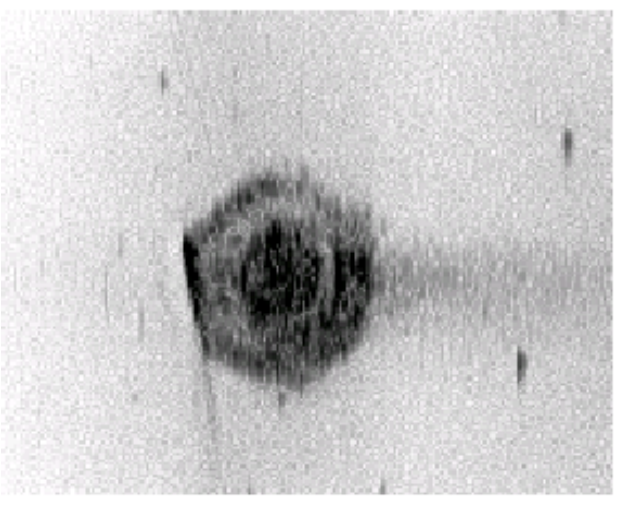

Here the long screw thread causes a shadow, which is visible as a blur to the right side of the thread.

The $2 \mathrm{D}$ character of the ultrasound wave beam suggests that images of large moving objects $(>35 \mathrm{~mm})$, or generally moving objects that are only partly inside the wave beam, may be interpreted as surface cross-section views. From a

Fig. (3). Metal screw thread with bolt, irradiated from the left side by ultrasound. Left panel: Bolt bottom. Right panel: Bolt top and thread with a shadowing effect. 
number of these partial images the shape and volume of the complete object could be reconstructed using interpolation or morphing techniques [10]. The possibility is strongly suggested by the images in Fig. (4) that shows three crosssection views of a polypropylene flat plate $(2 \mathrm{~mm}$ thick) with two distinctive features. Two notes are in order. First, since ultrasound will penetrate plastic material an image such as Fig. (4) also shows the back-wall of the plate. However, the medical imager does not recognise the difference in wave speed between water and plastic. Therefore the imaged thickness of the plastic plate will not be accurate. Second, the volume reconstruction is facilitated by the fact that most plastic waste particles are flakes and therefore practically flat (1-3 mm). This makes them float in more or less predictable horizontal orientation in the MDS ferrofluid after the main density separation, just before they are removed from the fluid. At the known flowing depth and preferred orientation, the particles can be observed from a favourable viewing angle by the ultrasound sensor array to minimise shadowing effects.

\subsection{Requirements to Throughput Measurement}

The accuracy of a particle volume measurement may be relaxed thanks to the large amount of particles involved in industrial MDS operation. The desired economical throughout for a PP/PE mixture is around $80 \mathrm{~kg} / \mathrm{min}$ of which $70 \%$ is PP that is to be separated into a pure stream [2]. This economical throughput entails 396000 PP particles per minute in the output stream when assuming an average particle size of $10 \times 2 \mathrm{~mm}$ (diameter $\mathrm{x}$ thickness). Using a conservative estimate, the ultrasound system may determine the volume of 2000 particles per minute. This number is based on the width of the sensor array $(35 \mathrm{~mm})$, the flow speed $(30 \mathrm{~cm} / \mathrm{s})$, a view on three particles at any given time, and a correction factor for viewing limitations. For throughput, the volumes of individual particles must be determined from the movie sequence of images using automated image processing. The quality of the images at hand will determine the error in the measured volume for each particle, which error may be assumed normally distributed. Since PP throughput will be presented as the total amount per minute, the relative accuracy of the throughput estimate will improve by a factor $\operatorname{sqrt}(\mathrm{N})$ where $\mathrm{N}$ is the number of average-size particles measured per minute. This means that at full capacity, when $\mathrm{N}=2000$, a measurement error of $45 \%$ for individual particles would still give a $1 \%$ accurate throughput estimate.

The capacity of the ultrasound system has further implications. The throughput measurement of 2000 particles covers only $0.51 \%$ of the $396000 \mathrm{PP}$ particles involved in economical MDS operation. However, if the measurements are performed on a representative sample of the total stream this ultrasound capacity will be sufficient. Possible stationary variations in throughput along the width of the flow channel may be measured and taken into account by allowing the sensor array to move so it can measure from different positions. These stationary variations in throughput may occur since the MDS is a flow system that can behave differently near the walls and outlets of the channel. If the MDS system would be designed as to fit this ultrasound capacity (2000 particles per minute/per $35 \mathrm{~mm}$ flow channel), the width of the MDS channel should be scaled up to $7 \mathrm{~m}$ to accommodate full economical throughput. At current projections it seems more realistic that future flow channels will not become wider than a few metres, which means in future the capacity of the ultrasound system should

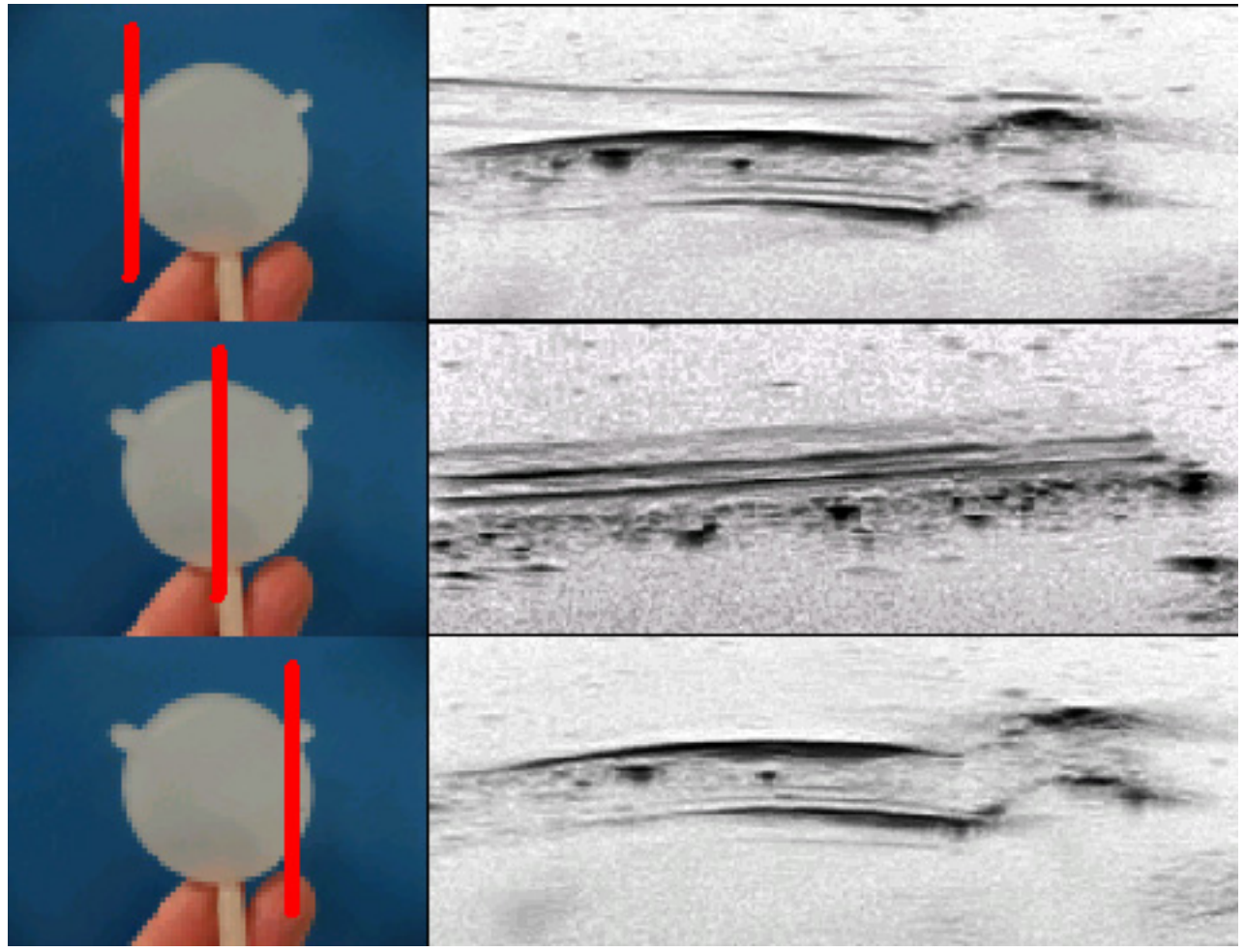

Fig. (4). From top to bottom three ultrasound cross-section images of a plastic plate, shown on the left side. The thick lines in the photos indicate the plane in which the wave beam was applied. 
at least be doubled. The first option is to work on the array viewing orientation and image processing so full use can made of the range of the sensor array, by which many more than the estimated three particles can be viewed at any given time. Technically, combining images of two or more sensor arrays is also an option, but this would require as many hardware systems and is not economical at present day costs. A better option would be to increase the flow speed of the ferrofluid in the MDS, since the real-time capability of the imager (25 images per second) is not yet fully employed. In fact, for the calculated capacity of 2000 particles each particle will be visible in 2 images. This redundancy improves the volume measurement accuracy, but perhaps it is better spend on doubling the capacity when the flow speed of the MDS is also doubled. A possible economical drawback of increasing the flow speed would be that the MDS channel and magnet have to be longer, making them more expensive, since in a faster flow the particles will take longer to reach a stable flowing depth.

\subsection{SAFT Imaging}

The physical principle of medical imaging is based on having a direct view of the object surface, and just like in an echo well experiment you need only to record the echo time to know the depth (distance). This is commonly called pulseecho testing and its principle is depicted in Fig. (5a).

Mathematically, it requires straight-forward multiplication of the data time coordinate by halve the water wave speed, since the waves travel up-and-down. This imaging method is efficient in data and computational requirements and explains the excellent real-time capability. The method proves particularly sensitivity to surface details over a relatively long range of up to several times the array aperture. However, it has two limitations. First, producing the optimum image under strongly changing conditions requires human intervention. The operator must change the orientation of the sensor array relative to the object for optimum view. Moreover, the operator must also regain optimum machine settings, such as illumination level, scanning mode, depth range, and dynamic range. In an MDS there may be little or no time to adjust the array or machine settings, i.e. the ultrasound system should mostly operate independent from the operator for the monitoring of plastics separation. Second, in the presence of strong reflectors the image may become distorted and difficult to interpret, for example if the walls or bottom of the MDS flow channel come into view. These distortions are commonly called ghosts, as the algorithm erroneously interprets these waves. In such cases an alternative imaging technique may prove useful as it may be better able to cope with the data at hand.

SAFT is based on another physical principle and uses the data in a different way. It is successful in non-destructive testing [4], but is also well suited for straight-forward imaging purposes. The basic principle of SAFT is wave interference, or actually the constructive and destructive interference of the logged sensor signals. Where the signals comply with waves originating from the same point the signals will summate constructively and produce a large response. The concept of waves originating from the same surface point and being captured by different sensor elements is depicted in Fig. (5b). Where the signals comply with waves that originated from different points, the phase differences will cause them to add up destructively and produce a small response. This physical principle makes SAFT more robust to strong wall and bottom reflections, since they will be suppressed by destructive interference. The effectiveness of SAFT depends on the available array aperture and improves with increasing viewing angle, which angle is illustrated in Fig. (5b). As a consequence, SAFT performs best if the object is relatively close to the sensor array where the viewing angle is largest.

Generally SAFT requires more data than the medical imaging technique for optimum performance, and the maximum data set from an array can be scanned by activating each individual sensor element as transmitter and receiver. For an array with $\mathrm{N}$ sensor elements this takes $\mathrm{N}^{2}$ measurements. An effective alternative, requiring less hardware effort, is to activate the elements individually in a sequential pulse-echo scan. This takes $\mathrm{N}$ measurements, but may give a lower image quality.

Wave beam forming is used in medical imagers to improve the directionality. It is technically achieved by activating several neighbouring elements at the same time, as illustrated in Fig. (5a). For example by activating $M$ elements the sequential scan with an N-element array takes $\mathrm{N}-\mathrm{M}+1$ measurements. It is noted that a SAFT data set could be used to conduct medical-type imaging, but not vice-versa because that data is at most a subset of the SAFT dataset. Besides data, SAFT generally requires more processing than the medical imaging principle. Therefore, to develop an industrially acceptable SAFT-based imaging system with (a)

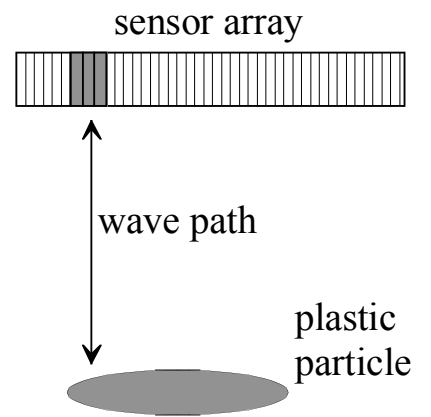

(b)

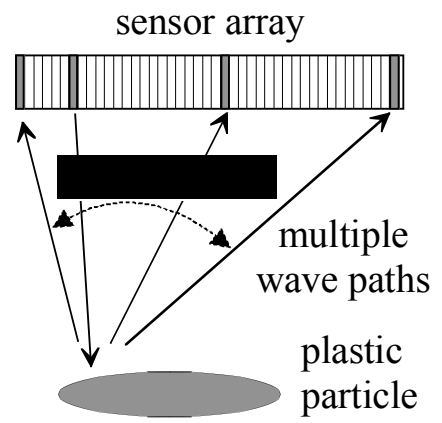

Fig. (5). (a) Straight wave paths used in medical imaging techniques; giving a simple relation between travel time and depth. (b) Multiple wave paths used in SAFT, allowing constructive summation of location-correlated signals. 
real-time capability may put even higher demands on the acquisition and processing hardware than a medical imager.

Both imaging principles may perform well after finding their own optimum array orientation and processing steps. To demonstrate, images are taken of the PVC object in Fig. (6) that has a circular and triangular indentation as easily recognisable features. A SAFT program was implemented in Matlab with the following elementary processing steps: data filtering, dynamic compression, SAFT kernel, Wiener filter, and nonlinear gray-level visualisation. The last two steps are basic image processing steps to enhance the surface contours and to suppress weak artefacts. The image processing is most likely better optimized in the software of the medical imager. It is noted that the SAFT kernel was a basic time-domain version, meant only to demonstrate the raw capability of the technique. Alternative versions of SAFT have been developed, for example Fourier domain approaches to reduce artefacts and/or reduce computational effort $[4,11]$. Fig. (7a) shows the SAFT image (128 pulse-echo measurements) and Fig. (7b) shows the image from the medical imager. The same $5 \mathrm{MHz}$ sensor array with 128 elements was used in both cases, but the SAFT data were collected on a different acquisition system.

The orientation of the array was slightly different in Figs. (7a, b), but the two imaging methods every time create their own peculiar small artefacts. The vertical surface parts in the SAFT image are quite faint because they hardly reflect any waves back to the sensor array. A solution would be to slightly tilt the array and scan these surface parts under a straighter angle. This would improve the viewing angle for the vertical surface parts, but probably reduce the visibility

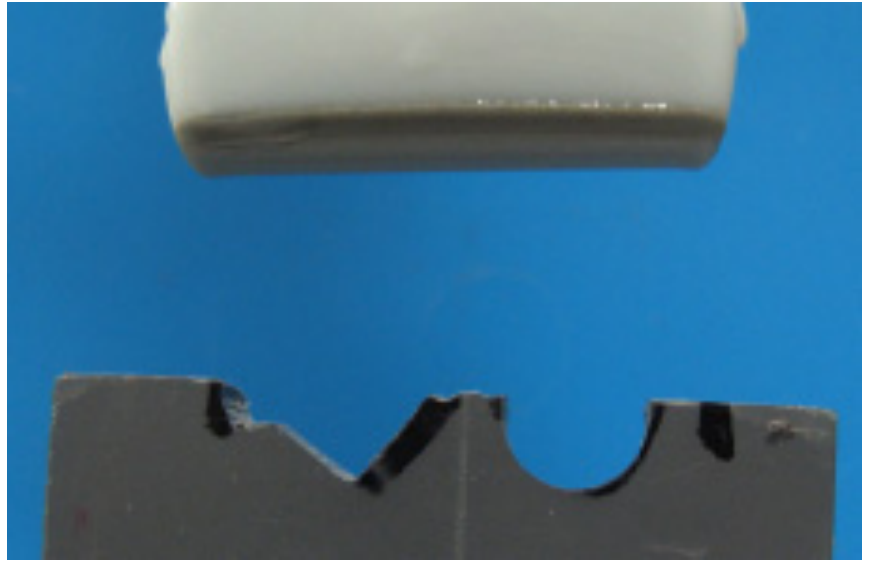

Fig. (6). Sensor array (top) and a PVC test object with recognisable features.

of other details. Also in Fig. (7a), the duplicated circular feature just below the actual circular indentation surface is a typical 3D artefact. It is a projection of scattered waves originating from the indentation edge parts just outside the ideal flat $2 \mathrm{D}$ plane of the array. This is caused by the fact that the array wave beam is still a few millimetres wide in the orthogonal direction, i.e. in the plane of the paper when looking at Fig. (6).

\section{MATERIALS AND METHODS}

\subsection{Polyolefins}

There are 100.000 polymer variants that all end up in waste. However, household waste and car shredder residue contain a lot of polypropylene (PP) and variants of

(a)

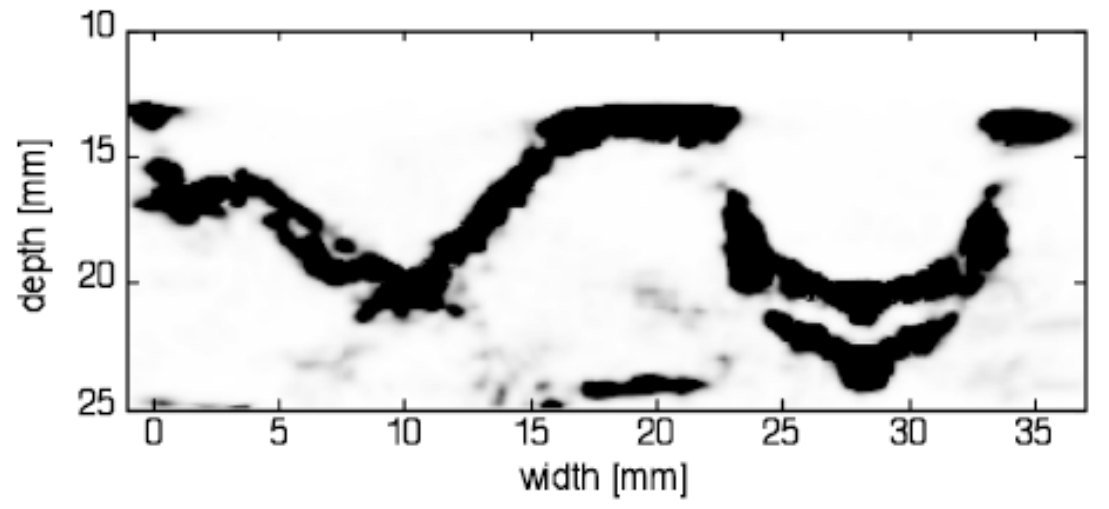

(b)

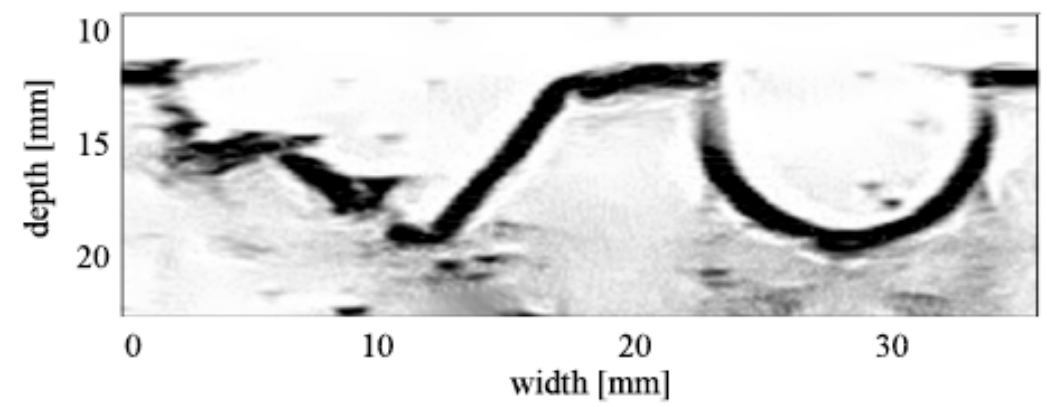

Fig. (7). Ultrasound image of test object in Fig. (6) for SAFT (a) and the medical imager (b). 
polyethylene (PE), which form the most important group for MDS processing. Specific types are low and high density polyethylene (LDPE, HDPE), linear low density polyethylene (LLDPE) and Ultra-high molecular weight polyethylene (UHMWPE). The output streams of a density separator like the MDS will contain impurities when the mass densities of different plastics are in the same range. On-line ultrasound inspection could detect these occurrences, provided their acoustic properties are distinctive [12].

\subsection{Experimental Set-Up}

Fig. (8) shows the calibrated experimental set-up for measuring acoustical properties. Plastic plates of thickness $0.5-3 \mathrm{~mm}$ were vertically immersed in the small water basin and subjected to a transmission measurement at a straight angle with respect to the plate. The transducers fired wave pulses of $5 \mathrm{MHz}$ centre frequency with $80 \%$ bandwidth. The acoustic wave speed $V$ of the plastic could be calculated from the difference in transmission travel time $T r$ with and without plastic sample as

$V=1 /\left(1 / V^{R}-T r / d\right)$

where $V^{R}$ is the water wave speed and $d$ the thickness of the sample.

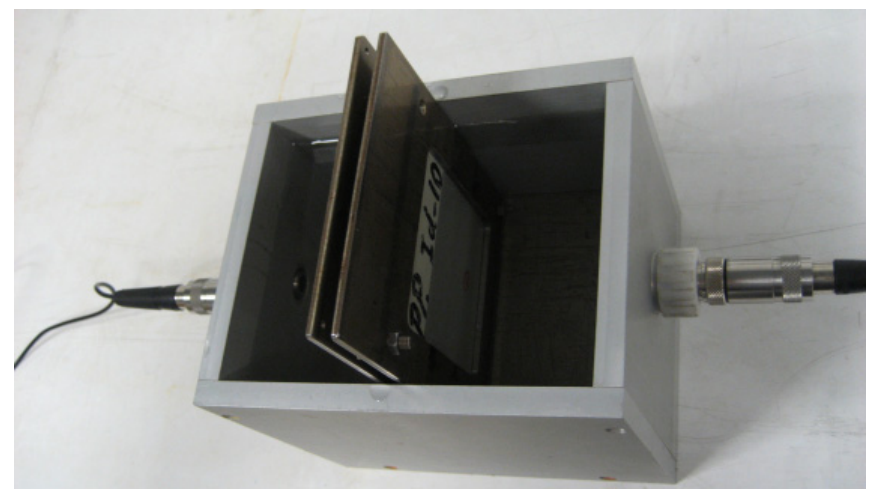

Fig. (8). Experimental set-up for plastics characterization.

\subsection{Acoustic Material Attenuation}

In the experiments the influence of shear waves inside the plastic could be neglected due to the fact that the waves are transmitted at practically straight angles through relatively thin samples. Each sample was flat and larger than the wave beam of the array, thus avoiding surface and edge scattering, and practically homogeneous and isotropic in its material properties. Under these conditions, without loss of generality, the acoustic attenuation in the present measurements may be investigated using a fluid model. The advantage is that it requires only two parameters (stiffness and attenuation), while it would be double for the elastic model. The applied 3D model is based on numerical integral evaluation of the Fourier-domain Green's function for acoustic waves propagating through multi-layered attenuating media [13]. In the present case there are only three layers: water-plastic-water. The semi-numerical approach, implemented in Matlab, also allows exact modelling of the wave diffraction due to the circularly shaped transducers visible in Fig. (8). The material attenuation is modelled by a first-order relaxation process that is introduced in a convolution-type constitutive equation: $\int k(t-\tau) p(\tau) d \tau=\nabla u$

$p$ is pressure, $u$ is displacement, and $k$ is the time-dependent compressibility of the plastic:

$k(t)=\frac{k_{0}}{2}(\boldsymbol{\delta}(t)+H(t) \exp (-t / \tau))$

$\boldsymbol{\delta}(t)$ and $H(t)$ are distributed functions known as the Dirac function and Heaviside step function, respectively, and $\tau$ is relaxation time. A longer relaxation time complies with stronger attenuation due to acoustic energy absorption. The attenuation was determined by matching the measured wave speed and calculated signal to the data obtained with the setup of Fig. (8). For improved accuracy the source wavelet was determined first, which is the effective pulse by which the piezoelectric transmitter is excited. For this purpose the signal of a calibration measurement (no plastic) was deconvoluted with the corresponding Green's function. This operation removes the influences of both propagation and transducer diffraction from the received signal and leaves the source wavelet as a result. Table $\mathbf{1}$ compiles the measured acoustic parameters for various plastic samples, including their mass densities that were determined with a Pico meter.

\subsection{Distinguishing Plastics Groups}

To visually distinguish different polyolefins the measured wave speeds are plotted against mass density. Additionally, it proves that the product of physical material stiffness and relaxation time is another parameter that plotted against mass density can help better distinguish plastic groups. The latter idea is based on the phenomenological inverse relation between the two parameters; if the stiffness of a certain plastic is decreased by some given thermal or chemical-physical process the acoustic attenuation usually increases, and vice versa. The relevant parameter will be referred to as "specific stiffness" and is denoted as

$$
S=\rho V^{2} \tau
$$

where $\rho$ is mass density, $\mathrm{V}$ is wave speed, and $\tau$ is relaxation time. Note that the first two terms of the previous formula present a physical material stiffness. The specific stiffness and wave speed are plotted against mass density in Figs. (9, 10). These show the tendency of the plastic types to form more or less isolated groups in the $\rho-\mathrm{S}$ and/or the $\rho-\mathrm{V}$ plane. Polypropylene (PP) tends to have the highest specific stiffness and in that respect it differs from low density polyethylene (LDPE), even though their mass density is in the same range. Also the reverse may happen. From Fig. (10) it proves that different plastics can have the same wave speed. However, as long as the mass densities are different there is no problem with identification inside an operational MDS, because the plastics will float at different depths and be distinctive through that aspect. Therefore, plastics identification and thus quality inspection on the basis of differences in acoustic properties appears feasible. Table $\mathbf{1}$ should in future work be extended with many more samples to accurately document the spread in acoustical properties for each polyolefin group. Using such a database, the boundaries between the acoustic properties attributed to 
Table 1. Acoustic Properties of Different Polyolefin Samples

\begin{tabular}{|c|c|c|c|c|c|}
\hline Sample Material & Density $\left[\mathrm{kgm}^{-3}\right]$ & Wave Speed $\left[\mathrm{ms}^{-1}\right]$ & Impedance [MRayl] & Relaxation Time [ns] & Attenuation@5MHz $[\mathrm{dB} / \mathrm{cm}]$ \\
\hline PP & 894 & 2540 & 2.27 & 5.3 & 43.8 \\
\hline PP & 895 & 2337 & 2.09 & 3.5 & 31.8 \\
\hline PP & 910 & 2671 & 2.43 & 3.6 & 29.0 \\
\hline PE & 1040 & 2094 & 2.18 & 1.4 & 14.3 \\
\hline PE & 951 & 2370 & 2.25 & 1.6 & 14.4 \\
\hline LDPE & 905 & 2174 & 1.97 & 2.5 & 24.5 \\
\hline LDPE & 905 & 2170 & 1,96 & 3.8 & 37.1 \\
\hline LLDPE & 906 & 2239 & 2.03 & 3.8 & 36.0 \\
\hline LLDPE & 906 & 2253 & 2.04 & 5.2 & 48.4 \\
\hline UHMWPE & 943 & 2338 & 2.20 & 1.6 & 14.6 \\
\hline UHMWPE & 943 & 2312 & 2.18 & 1.8 & 16.6 \\
\hline
\end{tabular}

specific groups can be decided and the overlap between them can be determined. The overlap means that a plastic material could be classified into two or more groups. The amount of overlap may be related to the achievable reliability of ultrasound quality inspection, as it determines the fractions of different polyolefins that apparently cannot be distinguished on the basis of acoustic properties alone.

\subsection{Requirements to Quality Inspection}

The operational MDS situation with a sensor array is different from the preceding calibrated set-up in mainly two aspects. First, the sensor irradiates the plastic from just one side, and secondly the plastics are not perfectly flat plates but irregularly shaped flake-like particles of $5-15 \mathrm{~mm}$ in size. The first aspect proves not a restriction, since the calibration measurements can also be carried out using a reflection technique. The second aspect creates uncertainty since surface scattering may obscure the desired materials information, and the desired information is mostly carried by waves that penetrated the interior of the particle. The method of data scanning determines the obtainable sensitivity of an inspection technique, while the size of the dataset will be decisive if the information is reliable. In principal there are only five unknowns for a particle that need to be identified simultaneously: wave speed, materials attenuation, mass density, and average (effective) particle thickness and diameter. The mass density may also be derived from the depth at which the particle is floating in the MDS ferrofluid. If imaging is used in conjunction with quality inspection the particle diameter and thickness may already be known also, which would leave just two unknowns to be estimated. The thickness divided by wave speed gives a travel time, or equivalently a phase delay, and is accompanied by amplitude information. By collecting many travel times and amplitudes under different angles of irradiation and receiving, a redundant set of equations can be generated from which the

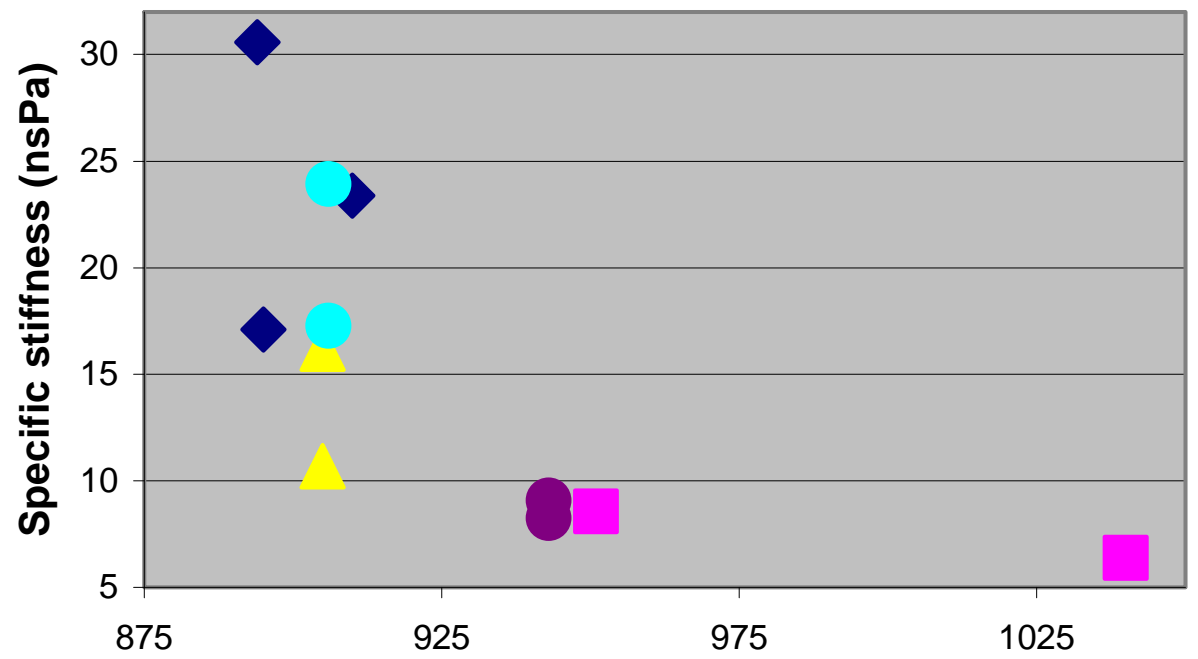

Mass density $\left(\mathrm{kg} / \mathrm{m}^{3}\right)$

\section{PP $\square$ PE $\triangle$ LDPE OLLDPE ○ UHMDPE}

Fig. (9). Specific stiffness against mass density for visual characterisation. 


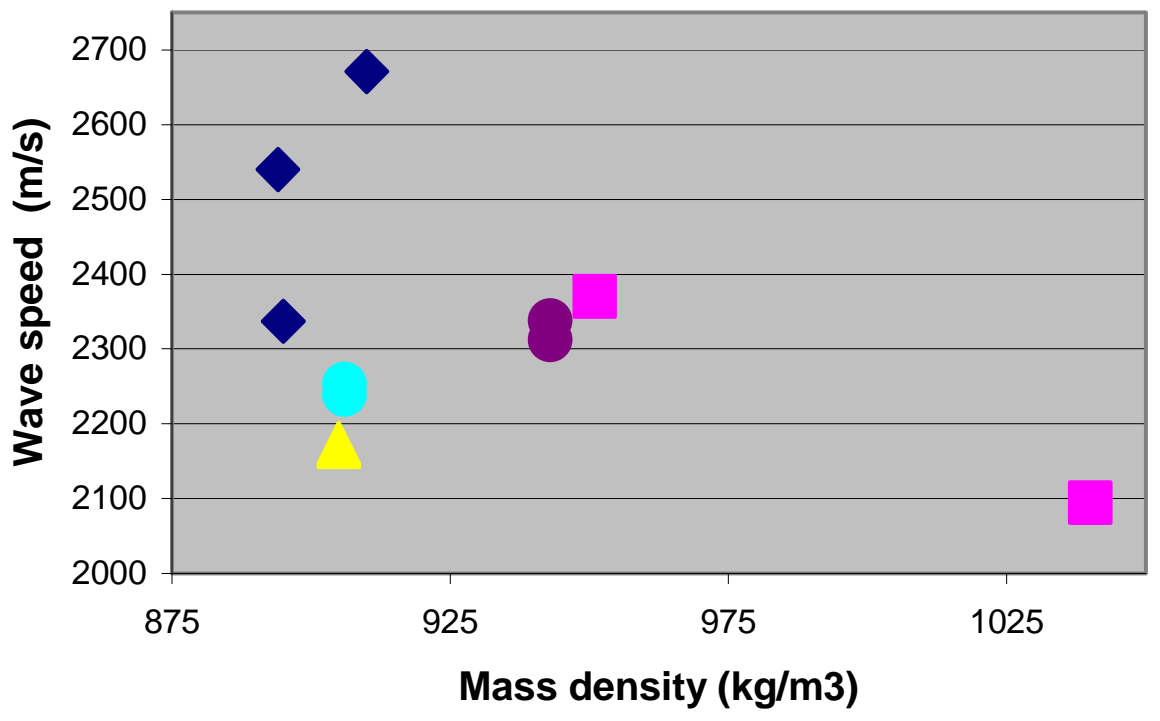

$\diamond \mathrm{PP} \square \mathrm{PE} \triangle \mathrm{LDPE} \bigcirc \mathrm{LLDPE} \odot \mathrm{UHMDPE}$

Fig. (10). Acoustic wave speed against mass density for visual characterisation.

unknowns could be solved, for example using a least-squares approach. The scanning method described for SAFT with $\mathrm{N}^{2}$ measurements would of course be suitable for this task, since it yields all the largest dataset that could possibly be obtained by an array. However, it would be preferable to find a quality inspection method that is more efficient in its data requirements and gives a better chance at affordable realtime scanning capability. The actual inspection technique and its data requirements are outside the scope of the present work.

Different from a common measurement method, quality inspection essentially knows two types of error; omission and false-call. These are best explained for the example of a stream of PP contaminated by LDPE. An omission is where a LDPE particle was not identified as such, and a false call is where a PP particle is mistaken for LDPE. Both types of error depend on the accuracy with which the inspection technique (dataset and processing) can measure the acoustic properties, and have minimum values depending on the distinctiveness of the acoustic properties belonging to different polyolefin groups.

The overall error in quality indication, denoted as $\Delta \mathrm{c}$, will be more tolerant to omissions than to false calls because the MDS is expected to perform well and keep contamination levels low. For example, if the contamination of a PP stream with LDPE is $5 \%$ at $5 \%$ chance on either error, the quality inspection would indicate $0.05 * 95+0.95 * 5$ $=9.5 \%$ LDPE $(\Delta \mathrm{c}=90 \%)$. In case of $5 \%$ LDPE and a $1 \%$ chance on false calls and $5 \%$ on omissions the quality indication would be $5.7 \%$ LDPE $(\Delta \mathrm{c}=14 \%)$, while a $5 \%$ chance on false calls and $1 \%$ on omissions would give a quality indication of $9.7 \%$ LDPE $(\Delta c=94 \%)$. It may be clear from these examples that to some degree the two types of error may even cancel each other. Therefore the performance of the quality inspection technique is best determined in a few tests using an input stream with known contamination contents. The minimum to be detected contamination is about $1 \%$ [2], where an overall error of $\Delta \mathrm{c}=25 \%$ seems acceptable.

So far it was assumed that all particles passing through the beam of the sensor array are uniquely identified. Under operational conditions there could be a small fraction of particles that cannot be acoustically identified as part of just one group, e.g. they could be both PP and LDPE. In principle this causes a third type of error for quality inspection, provided it can be proven by different means than ultrasound that the plastics in question do belong to a specific group. In that case the third type of error may also be specified through performance tests.

Contrary to throughput measurement, the overall error for quality inspection does not depend on how many particles are identified per minute, i.e. the inspection capacity plays no role in the performance. But where it concerns the MDS economical capacity and stream sampling the implications are the same as for throughput measurement. For a given size MDS with a build-in ultrasound inspection capability the maximum throughput that could be accommodated by the MDS is probably limited by the capacity of the inspection system. Also, the single sensor array in quality inspection will cover only a small fraction of the amount of particles processed under economical MDS operation. Therefore similar considerations apply as for throughput inspection where stream sampling is concerned. The real-time capability of a quality inspection system will require some attention due to the larger dataset and need for more elaborate processing than for throughput analysis.

\section{CONCLUSIONS}

Commercial medical imaging systems have potential for monitoring the separation process of waste Polyolefin particles in Magnetic Density Separation (MDS). The offthe-shelf medical imager also has excellent real-time capability and allows monitoring from inside the ferrofluid flow for particle speeds (flow speed) at least up to $30 \mathrm{~cm} / \mathrm{s}$. 
The system may also allow for throughput measurement provided the amount of particles passing through the sensor array beam does not exceed 2000 particles per minute. Both monitoring and throughput measuring capability may be achieved, provided the optimum viewing conditions of the sensor array remain stable. In case the image quality diminishes, an alternative imaging method could be employed to act in conjunction with the medical imaging technique. For this purpose the SAFT method is proposed that is based on a different physical principle. Since SAFT requires a larger dataset than the medical imaging technique it would put higher demands on the hardware to achieve realtime capability. Ultrasound also has potential for quality inspection using a sensor array inside the ferrofluid of the MDS. It has been shown that different groups of polyolefins are acoustically distinctive, which is the primary condition for ultrasound quality inspection. In throughput measurement the performance will improve with the inspection capacity of the ultrasound system. This is different for quality inspection, which performance depends only on the accuracy with which the inspection technique identifies different plastics. Both quantitative techniques will employ a single sensor array, which limited size can cover only a fraction of the total amount of particles processed in operational MDS. If the stream sampling is representative this is not a limitation for these techniques. Moreover, possible stationary variations in either throughput or quality across the full width of the flow channel may be accommodated by allowing the sensor array to be moved.

\section{ACKNOWLEDGEMENTS}

The study was developed thanks to the financial support of the European Commission in the framework of the FP7 Collaborative project "Magnetic Sorting and Ultrasound
Sensor Technologies for Production of High Purity Secondary Polyolefins from Waste (W2Plastics)". Grant Agreement No. 212782.

\section{REFERENCES}

[1] Website of W2Plastics, 2008: http://www.w2plastics.eu

[2] E. J. Bakker, P. C. Rem and N. Fraunholcz, "Upgrading mixed polyolefin waste with magnetic density separation", Waste Manage., vol. 29, no. 5, pp. 1712-1717, December 2009.

[3] D. J. K. Dowsett, A. Patrick and R. E. Johnston, "The Physics of Diagnostic Imaging", Hodder Arnold, London, 2006.

[4] K. J. Langenberg, "Synthetic aperture focusing technique signal processing”, NDT Int., vol. 19, no. 3, pp. 177-189, January 1986.

[5] R. A. Marklein, "Linear and nonlinear inversion algorithms applied in nondestructive evaluation", Inverse Problems, vol. 18, no. 6, pp. 1733-1759, October 2006.

[6] G. Bonifazi, "Innovative sensing technologies applied to postconsumer polyolefins recovery", Metalurgia Int., vol. 14, no. 2, pp. 5-10, January 2009.

[7] S. Karlsson, "Recycled polyolefins. Material properties and means for quality determination", Adv. Polym. Sci., vol. 169, pp. 201-229, February 2004

[8] G. D. Ludwig, "The velocity of sound through tissues and the acoustic impedance of tissues", JASA, vol. 22, no. 6, pp. 862-866, May 1950.

[9] A. Józefczak and A. Skumiel, "Acoustic and magnetic properties of a dense commercial magnetic fluid", Czechoslovak J. Phys., vol. 54, no. 4, pp. D647-D650, July 2004.

[10] P. Kilian, "New visualization tools: computer vision and ultrasound for MIS navigation", Int. J. Med. Robotics. Comput. Assist. Surg., vol. 4, pp. 23-31, January 2008.

[11] A.J. Hunter, "The wavenumber algorithm for full-matrix imaging using an ultrasonic array", IEEE Trans. Ultrason. Ferroelectr. Freq. Control, vol. 55, no. 11, pp. 2450-2462, May 2008.

[12] J. B. Hull, "Characterisation of polymers in mixed waste streams using ultrasonic broadband attenuation analysis", Mechanical and corrosion properties. Series A, Key engineering materials, vol. 99100 , pp. 57-64, 1995.

[13] B. L. N. Kennett, "Seismic Wave Propagation in Stratified Media", Cambridge, New York, Cambridge University Press, 1983. 